\title{
Droperidol: An effective adjuvant for difficult cases of conscious sedation?
}

\author{
Eric Semlacher MD FRCPC
}

\section{ARTICLE}

Cohen J, Haber GB, Dorais JAL, et al. A randomized, double-blind study of the use of droperidol for conscious sedation during therapeutic endoscopy in difficult to sedate patients. Gastrointestinal Endoscopy 2000;51(5):546-51.

$S_{\text {then }}^{\text {mans }}$ truggling with a 'difficult to sedate patient' during a therapeutic endoscopic intervention, such as for massive gastrointestinal bleeding or removal of a foreign body, detracts from the quality of the intervention and potentially places the patient at increased risk. General anesthesia or propofol are options for sedation in such patients. However, these typically require the presence of an anesthesiologist, which can sometimes be a challenge to coordinate and significantly increases the total procedure costs. Thus, a safe sedation alternative would obviously be welcomed. The stated aim of the study by Cohen et al was to determine whether adding droperidol to standard benzodiazepine and narcotic regimens improves the quality of conscious sedation for prolonged or therapeutic endoscopy procedures in patients who are anticipated to be difficult to sedate.

Ninety-eight patients were randomly assigned to receive placebo (saline) or droperidol (Inapsine, Janssen Pharmaceutical, Canada) $(5 \mathrm{mg}$ ) along with the conscious sedation regimen of midazolam and meperidine in doses adjusted by the endoscopist to the individual case. One-half of the droperidol (or placebo) dose was administered at the time of the initial midazolam/meperidine administration, with the second half given 2 min later. Enrolled patients were 18 years of age or older who abused narcotic, benzodiazepine or alcohol, or who had known previous sedation difficulties. They certainly appeared to be 'difficult to sedate', judging from the report that more than $25 \%$ of endoscopies performed in the placebo group were rated by the endoscopy staff as being more difficult due to sedation, and in $30 \%$ to $50 \%$ of placebo cases, the sedation was rated as poor. The procedures done were either endoscopic retrograde cholangiopancreatography $(83 \%)$ or therapeutic upper gastrointestinal endoscopy (17\%). The protocol blinded all participants during the endoscopy procedure and obtained impressions of sedation from each participant.

The design and implementation of this study appeared to be satisfactory. It is possible that better outcomes might have been achieved if droperidol were used as an intravenous or intramuscular premedication given 30 min before starting the conscious sedation (see drug information below) rather than simply as an immediate adjunct. This would have been my main recommendation for modification to the study protocol. Nonetheless, despite this potentially confounding feature, positive results were still identified. A $10 \%$ reduction in the time to starting the procedure and reductions in drug requirement (23\% less midazolam and $15.6 \%$ less meperidine) were noted in the droperidol group. These reductions were statistically significant, but I think that their true clinical relevance could be debated. What was more important was the observation by the blinded participants that sedation appeared to be clearly improved by the addition of droperidol. Overall ratings of sedation quality by the endoscopy staff were higher in the droperidol group, the percentage of procedures with poor ratings was about one-half that of the placebo group, and the percentage of 
cases in which sedation-related interruptions occurred was reduced from $32.6 \%$ to $12.8 \%$. Time for recovery and frequency of complications of sedation were not significantly different between the placebo and droperidol groups.

Droperidol is a neuroleptic butyrophenone tranquilizer with some sedative and antiemetic effects. Use of droperidol alone for sedation is not recommended. The drug is rapidly absorbed following intramuscular injection, and the therapeutic effect is noted within 3 to $10 \mathrm{~min}$. However, its peak effect is not achieved until $30 \mathrm{~min}$ or more after either intramuscular or intravenous administration. Sedative effects following a single intravenous or intramuscular dose typically last 2 to $4 \mathrm{~h}$. The most common adverse effects are hypotension and tachycardia, which tend to be self-limited and minor. Nonetheless, careful attention should be paid to the general principle of assuring that the patient is adequately hydrated before endoscopic sedation. Droperidol is structurally similar to haloperidol and shares the toxic potentials of phenothiazines. For procedures without general anesthesia (such as endoscopy), the recommended adult dose is 2.5 to $10 \mathrm{mg}$ intramuscularly 30 to $60 \mathrm{~min}$ before the procedure followed by an additional 1.25 to $2.5 \mathrm{mg}$, usually intravenously, if needed, during the procedure. The drug is metabolized in the liver and excreted primarily in the urine $(75 \%)$ and feces $(22 \%)$. Therefore, dosage reduction does need to be considered for patients with significant hepatic and possibly also renal dysfunction. Because elderly or debilitated patients appear to be prone to more severe and/or prolonged complications when they occur, dosage reduction is also suggested for these patient populations.

Use of droperidol in combination with narcotics and benzodiazepines to achieve conscious sedation for endoscopic procedures has been evaluated previously. Significant benefits have not always been demonstrated, perhaps because sedation for the average patient and for uncomplicated, diagnostic, elective endoscopic procedures typically poses no great problems. Cohen et al make an important contribution to the literature in showing the value and apparent safety of droperidol as an adjunct to conscious sedation in 'difficult to sedate' patients. This has been my experience with the drug also. The cost of droperidol was listed as approximately US $\$ 5$ for $5 \mathrm{mg}$, which is not a great addition to the overall cost of the endoscopic procedure. Bearing in mind the precautions and principles of use mentioned earlier, droperidol is worth considering when good patient sedation is crucial to the endoscopy, but there is suspicion that this might not be achieved, owing to the clinical setting or to patient-related variables. 


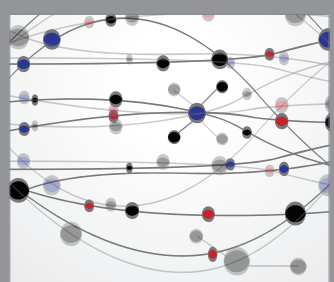

The Scientific World Journal
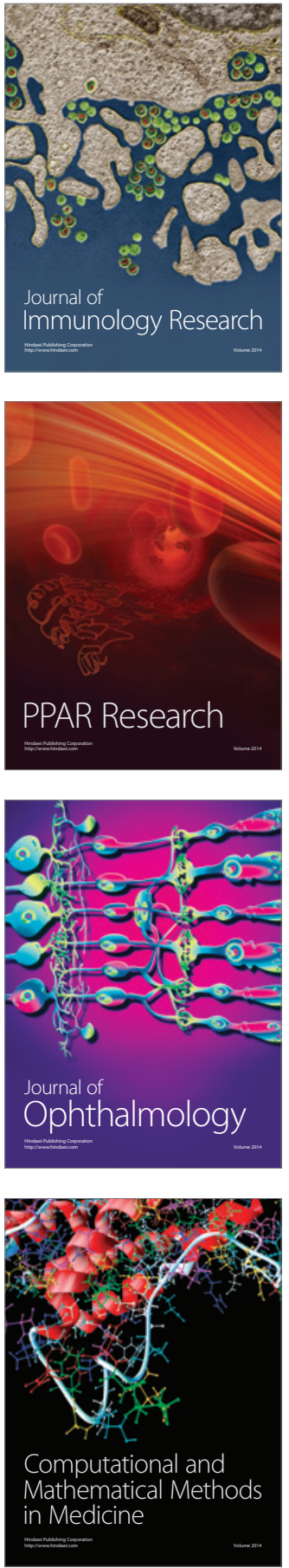

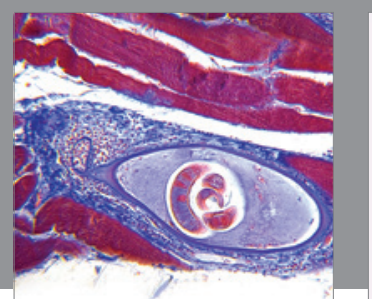

Gastroenterology Research and Practice

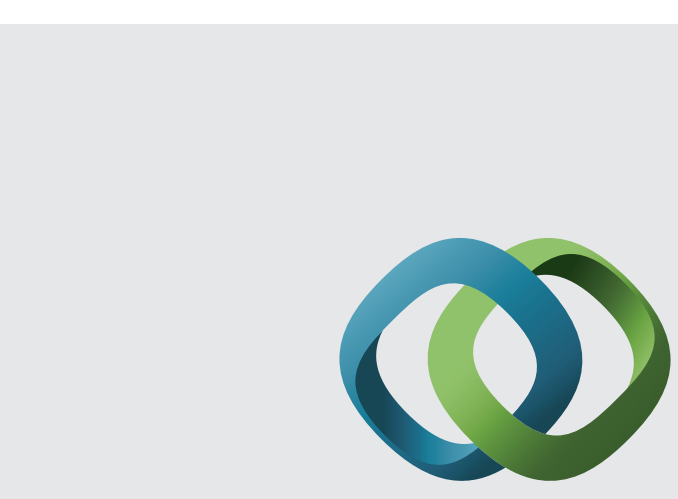

\section{Hindawi}

Submit your manuscripts at

http://www.hindawi.com
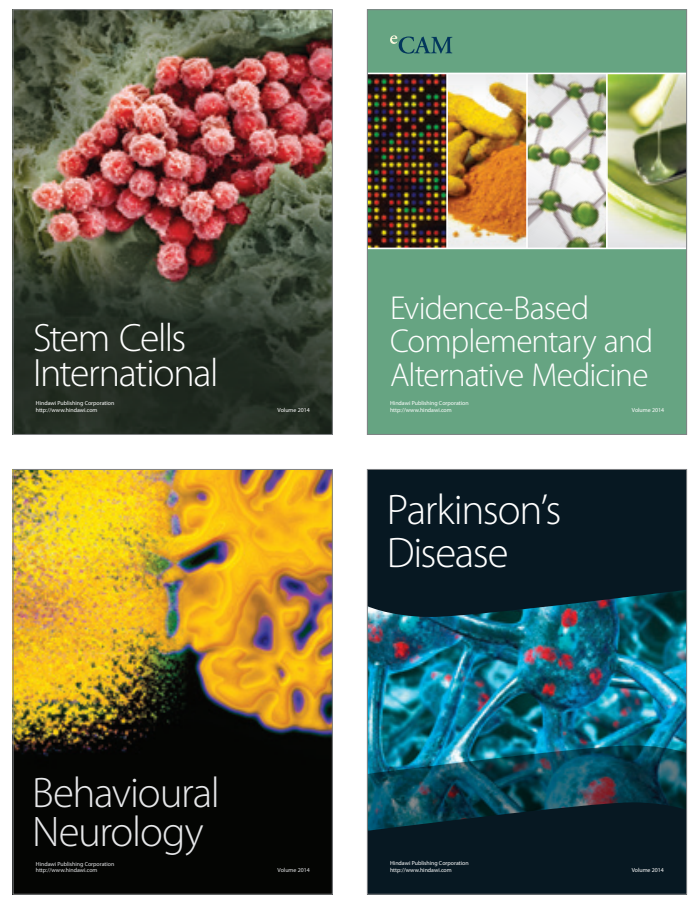
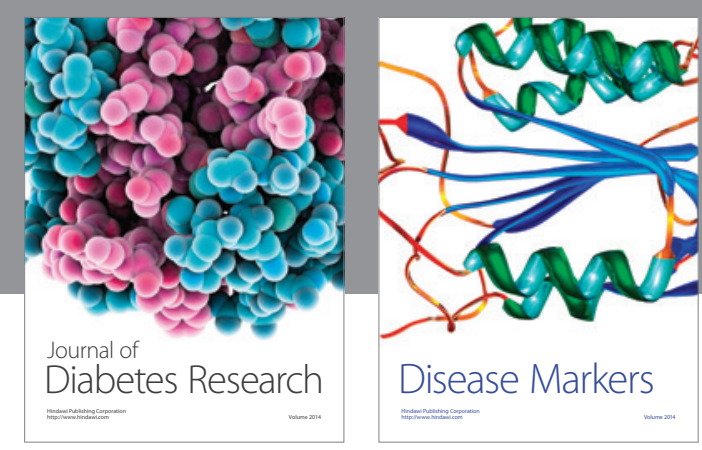

Disease Markers
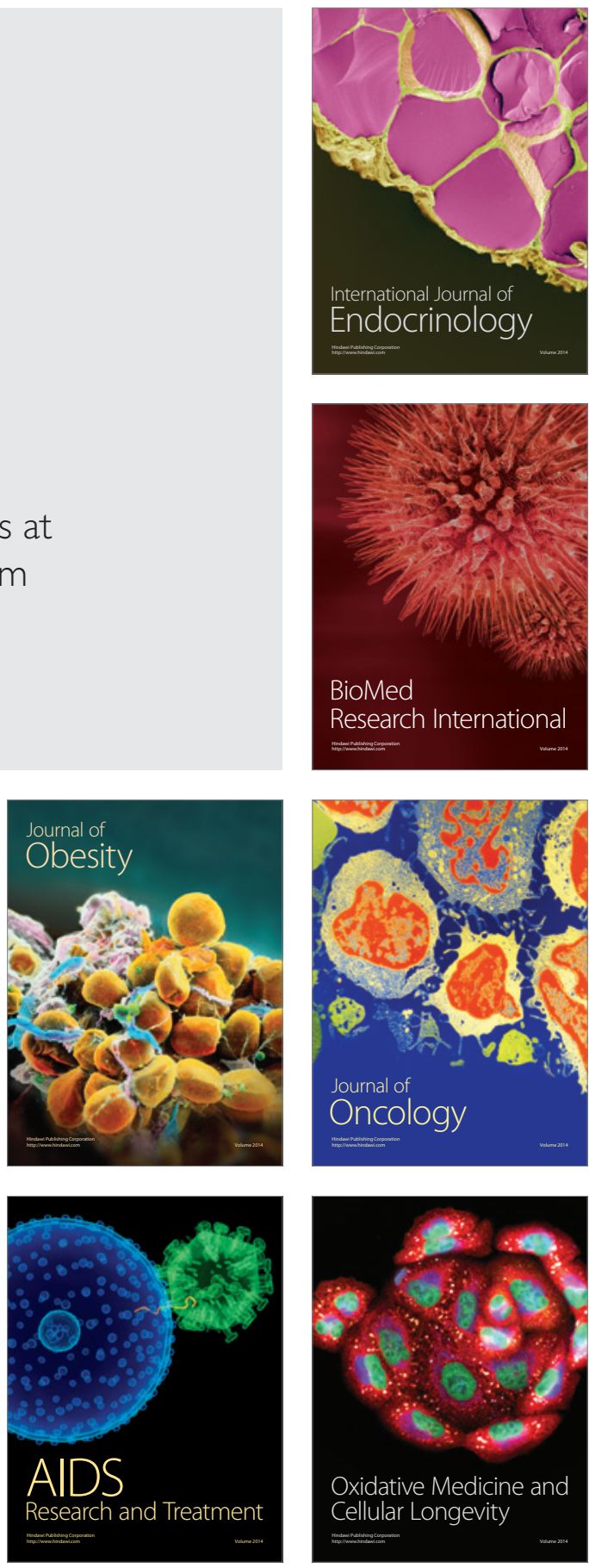